\title{
Die Struktur der Pachytänchromosomen einiger Acanthaceen, sowie eine Reihe neu bestimmter Chromosomenzahlen ${ }^{1}$
}

\author{
Yoshiro Takizawa ${ }^{2}$
}

Aus dem Botanischen Institut der Universität Freiburg i.Br., Deutschland

Eingegangen am Februar 1. 1957

\section{Einleitung}

Die Ordnung der Tubifloren fand bisher auf cytologischem und cytogenetischem Gebiet besondere Beachtung. Allein im hiesigen Institut wurden folgende systematische Gruppen bearbeitet: Gesneriaceen (Oehlkers 1944, Eberle 1956), Digitalis-Arten (Linnert 1949), Solanaceen (Gottschalk 1954) und Salvia-Arten (Linnert 1955). Als typisches Merkmal fand sich bei den vorwiegend in der Meiosis beschriebenen Chromosomen eine starke Heterochromasie. Als schwierig zu beantworten erwies sich die Frage nach der Chromosomengrundzahl der einzelnen systematischen Gruppen. Es war das Ziel der vorliegenden Arbeit, diese Verhältnisse bei den cytologisch wenig bearbeiteten Acanthaceen zu unterschen.

\section{Material und Methode}

Die verwendeten Pflanzen sind ausschließlich solche, die im Warmhaus der Freiburger Gewächshäuser gezogen wurden. Die Untersuchung der Meiosis wurde in den 今 Gonotokonten (PMZ) vorgenommen. Um eine rasche und vollständige Fixierung zu gewährleisten, wurden die Antheren vollständig freipräpariert und dann in Carnoy (Alkohol: Eisessig $=3: 1$ ) gebracht. Angefärbt wurde in gesättigter Carminessigsäure mit Eisenzusatz (2.5 g Carmin/ $1000 \mathrm{ccm} \mathrm{50 \%} \mathrm{ige} \mathrm{Essigsäure;} 0.5 \mathrm{ccm} \mathrm{10 \%} \mathrm{ige} \mathrm{FeCl}_{3}$-Lösung) in flachen Petrischalen. In Tabelle 1 sind die Planzen, bei denen wir Ergebnisse erzielen konnten, zugleich mit den neubestimmten Chromosomenzahlen angeführt.

\section{Empirischer Teil}

\section{Die neubestimmten Chromosomenzahlen einiger Acanthaceen}

1 Es ist dem Autor eine angenehme Pflicht, Herrn Prof. Dr. F. Oehlkers, welcher mir ein Thema aus seinem Arbeitsgebiet überließ und einen Arbeitsplatz mit Mikroskop zur Verfügung stellte, herzlichst zu danken. Herrn Dr. Eberle danke ich für die Einführung in die cytologische Arbeitsrichtung und die Hilfe bei der Anfertigung der Fotos und der Niederschrift obiger Arbeit. Der Deutsche Akademische Austauschdienst und die Alexander von Humboldt-Stiftung, sowie die Wissenschaftliche Gesellschaft zu Freiburg i.Br. ermöglichten durch Studienstipendien meinen Aufenthalt in Deutschland, wofür ich ebenfalls danke.

2 Professor, Hokkaido Universität, Sapporo, Japan. 
Es fanden sich folgende Haploidzahlen : 9, 10, 12 ?, 14, 16, 17, 18, 21. Keine der genannten Zahlen erscheint besonders gehäuft. Eine gemeinsame

Pflanzennamen

Thunbergia alata

Strobilanthes isophyllus

Hemigraphis repanda

Ruellia devosiana

Aphelandra aurantiaca

Aphelandra chamissoniana

Peristrophe salicifolia

Hypoesthes phyllostachyi

Thyrsacanthus rutilans

Fittonia argyroneura

Fittonia gigantea

Fittonia verschaffeltii

Justitia coccinea

Belloperome guttata

* Darlington 1935
$n$-Zahl

9*

10

14

17

14

14

21

11-13

21

18

18

18

16

14

Chromosomengrundzahl aufzustellen ist jedoch auf Grund von Zahlenangaben nicht möglich, weil nur unter Berücksichtigung der Chromosomenstruktur eine solche Spekulation einigermaßen $z u$ verantworten ist. Diesser Frage soll gegebenenfalls später nachgegangen werden (Abb. 1).

2. Die Struktur der Pachytänchromosomen

I. Thunbergia alata $(n=9)$

Sämtliche Chromosomen verfügen über größere Mengen von Heterochromatin, welches besonders auffällig beiderseits der Insertionsstellen liegt. Außer dieser lokalen Anhäufung sind kleinere Mengen von Heterochromatin in die dadurch stärker anfärbbaren euchromatischen Enden eingelagert. Mit den gegebenen Chromosomenlängn von 50 $-70 \mu$ ist es unmöglich, diese Maße zur Identifikation der ein-

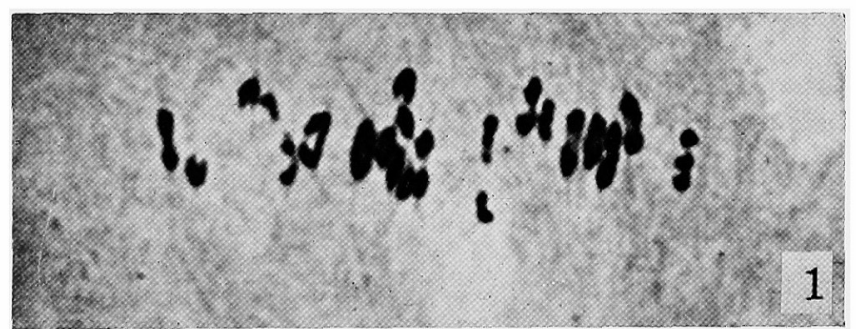

Abb. 1. Ruellia devosiana. Met. I. $n=17 ; 2000: 1$ zelen Chromosomen zu verwenden. Die Lage der Insertionsstellen in den einzelnen Chromosomen ist unterschiedlich; meist liegt eine mediane bis submediane Insertion vor. Ein Teil der Chromosomen läßt sich mittels charakteristischer Heterochromasie und der Lage der Insertionsstellen identifizierbar beschreiben.

II. Strobilanthes isophyllus $(n=10)$

In diesem Genom sind die heterochromatischen Anteile und Insertionsstellen auffallend abwechslungsreich verteilt, sodaß es nicht schwer fällt, die einzelnen Chromosomenpaare zu identifizieren. Sie haben Längen zwischen 20 und $41 \mu$. In der nachfolgenden Beschreibung werden die kaum wahrnehmbaren Mengen Heterochromatin, welche die dunkle Färbung und Spiralisation der sogenannten euchromatischen Chromosomensegmente bedingen, nicht berücksichtigt (Abb. 2 und 3).

Chromosom Nr. I ist als Nukleolenchromosom das längste des Genoms $(41 \mu)$. Satellit und Insertionsstelle bleiben unzugänglich (ungefähr 1/3 des Gesamtchromosoms!). Úber die übrigen Chromosomenregionen finden sich in großen und verschiedenen Abständen 10- 
14 heterochromatische Chromomeren verteilt. - Bei Chromosom Nr. II $(36 \mu)$ ist die submedian liegende Insertionsstelle von zwei größeren Heterochromatinblöckchen (je 2-3 Chromomeren entsprechend) begrenzt; in deren Nähe findet man beiderseits locker verteilt einige normal große Chromomeren. Mit diesem Chromosom ist Nr. X vergleichbar, welches viel kürzer ist $(20 \mu)$. Dessen submedian liegende Insertionsstelle wird durch geringere Mengen Heterochromatin begrenzt.

Die Chromosomen $\mathrm{Nr}$. IV und IX haben subterminal liegende Insertionsstellen, welche
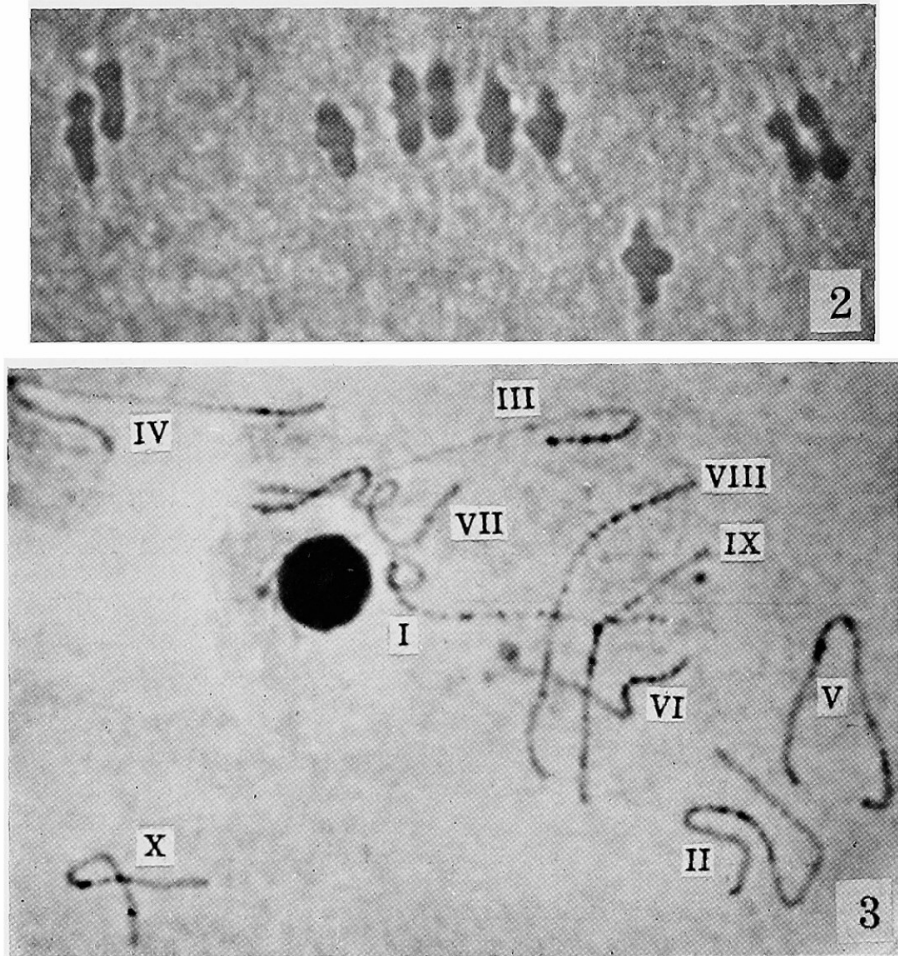

Abb. 2 u. 3. 2. Strobilanthes isophyllus. Met. I. $n=10 ; 2000: 1$.

3. Strobilanthes isophyllus. Pachytän. $n=10 ; 2000: 1$ beiderseits gleiche Mengen Heterochromatin (bei Nr. IV je 2 Makrochromomeren und ca. 2 Mikrochromomeren; bei Nr. IX je 1 Mackrochromomer und ein Mikrochromomer) aufweisen. Ihr eines euchromatisches Chromosomenende ist äußerst kurz, das andere sehr lang.

Als einziges Chromosom ist $\mathrm{Nr}$. $\mathrm{V}$ median inseriert. Außer dem Heterochromatin an den Insertionsstellen findet sich submedian eine Anhäufung von 3 Chromomeren.

Die Chromosomen Nr. III, VI, VII und VIII haben nur ein langes euchromatisches Ende und größere Mengen von Heterochromatin, welches an beiden Seiten der Insertionsstelle in charakteristischer Weise angeordnet ist. Die Insertionsstellen liegen bei Nr. III und VI subterminal, bei Nr. VII und VIII submedian. Chromosomenlängen: 35, 28,28 und $27 \mu$.

\section{Aphelandra aurantiaca $(n=14)$ und A. chamissoniana $(n=14)$}

Von beiden Arten gelang es, in dem einen Fall 10, im anderen 8 Pachytänchromosomen morphologisch zu erfassen. Sowohl in der Chromosomenstruktur als auch in den Chromosomenlängen (26-42 $\mu$ ) finden sich große Ähnlichkeiten. Auffallend ist die sehr starke Heterochromasie; so sind die meist median bis submedian liegenden Insertionsstelen beiderseits häufig von 4-7 Makrochromomeren starken Heterochromatinblöckchen begrenzt (Abb. 4, 5 und 6). Selten erscheinen in den ebenfalls auffallend spiralisierten euchromatischen Chromosomenenden größere lokale Anhäufungen von Heterochromatin, welche die Identifizierbarkeit erleichtern könnten. Möglicherweise sind zwei. Satellitenchromosomenpaare gegeben; beide besitzen einen großen heterochromatischen Trabanten. 
IV. Thyrsacanthus rutilans $(n=21)$

Es gelang, 12 der 21 Chromosomenpaare morphologisch zu erfassen. Alle Chromosomen sind in sämtlichen Regionen stark spiralisiert. Es ist bemerkenswert, daß es kaum möglich ist, eine Grenze zwischen euchromatischen und heterochromatischen Chromosomenanteilen zu ziehen. Dies ist dadurch bedingt, da $B$ sich die mächtigen heterochromatischen Anteile nicht nur auf die Regionen beiderseits der Insertionsstellen beschränken, sondern $\mathrm{da} B$ die Menge des Heterochromatins, bei der Insertionsstelle beginnend, gegen die Chromosomenenden hin kontinuierlich abnimmt. Da-
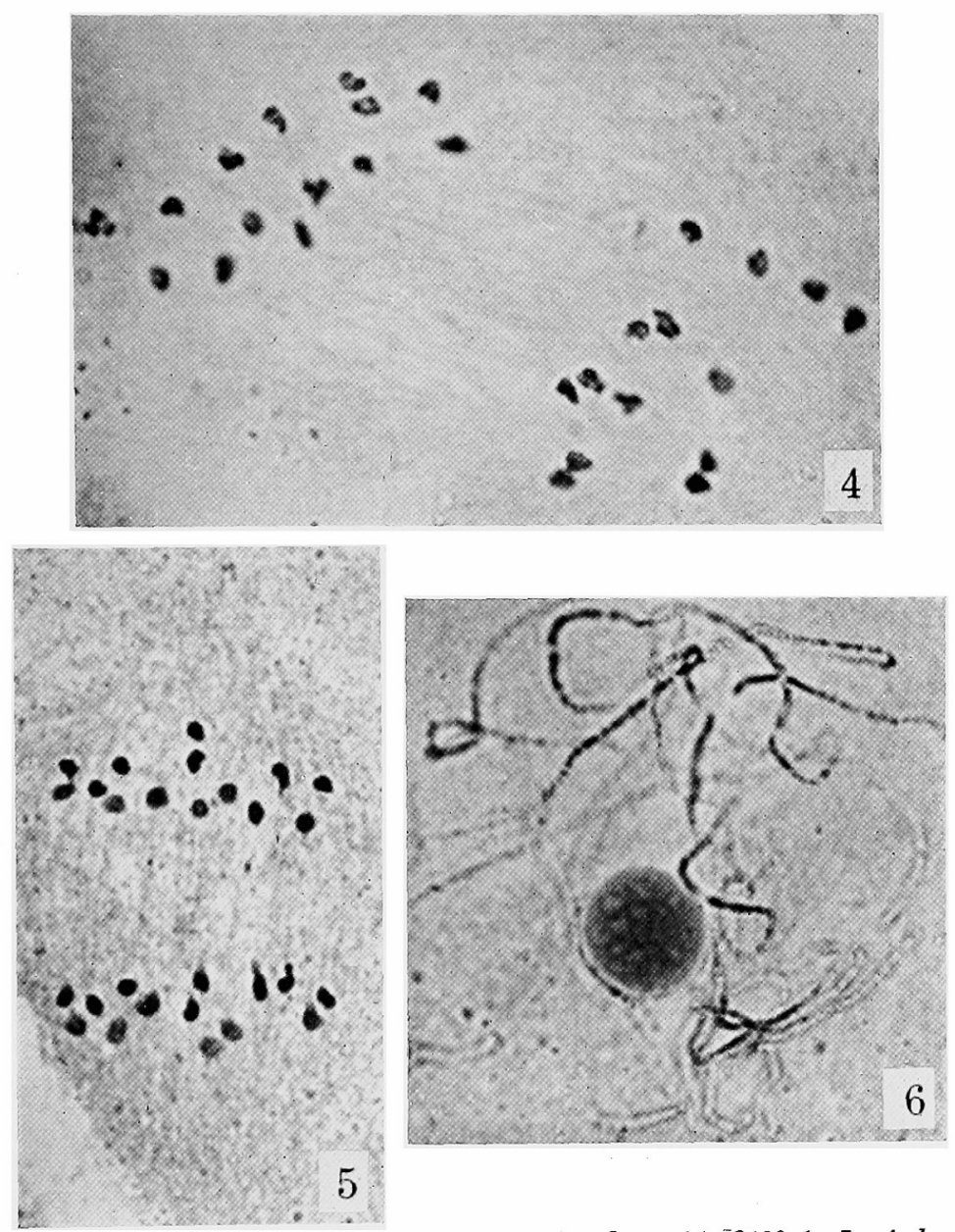

Abb. 4-6. 4. Aphelandra aurantiaca. An. I. $n=14 ; 2400: 1$. 5. Aphelandra chamissoniana. An. I. $n=14 ; 1200: 1$. 6. Aphelandra awrantiaca. Pachytän. $n=14 ; 1200: 1$. durch besitzen die sogenannten euchromatischen Chromosomenenden größere Partikel von Heterochromatin, welches auch den hohen Spiralisationsgrad bedingt. Außer dem Nukleolenchromosom sind sämtliche erfaßten Chromosomen median bis submedian inseriert. Die gemessenen Längen betragen 25-39 $\mu$ (Abb. 7 und 8).

V. Fittonia argyroneura, $F$. gigantea, $F$. verschaffeltii $(n=18)$

Für alle drei Arten von Fittonia gilt hinsichtlich der Chromatine und der spiralisation das gleiche, wie wir es oben für Thyrsacanthus beschrieben haben. Es war möglich, bei $F$. argyroneura 13 , bei $F$. gigantea 10 und bei $F$. verschaffeltii 14 Chromosomen vollständig zu beschreiben. Die 


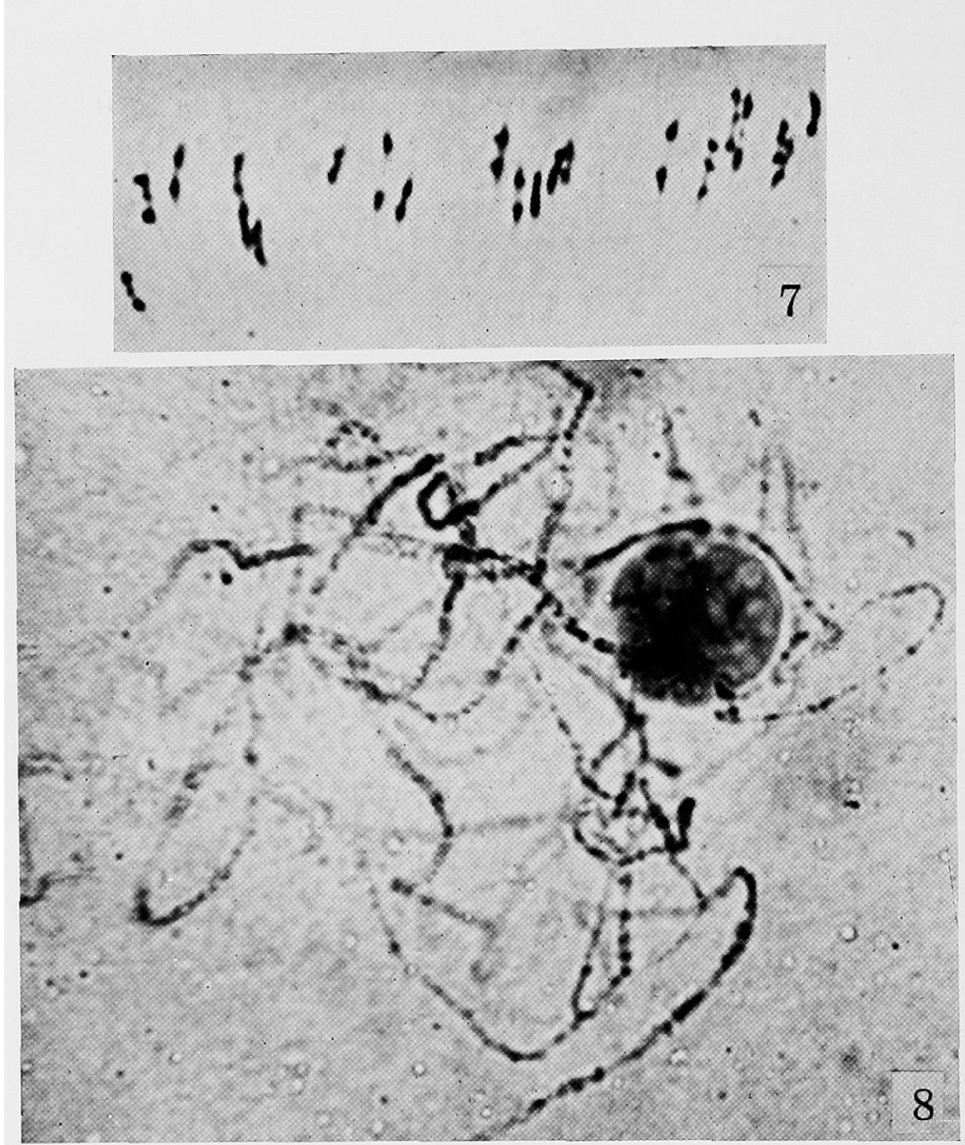

Abb. 7 u. 8. 7. Thyrsacanthus rutilans. Met. I. $n=21$; 2000:1. 8. Thyrsacanthus rutilans. Pachytän. $n=21 ; 2000: 1$.
Verteilung von $\mathrm{Eu}$ - und Heterochromatin ist ebenfalls mit den Verhältnissen bei Thyrsacanthus vergleichbar (Abb. 9). Lediglich die Beladung der euchromatischen Enden mit Heterochromatin ist bei den FittoniaArten schwächer. Aus diesem Grunde ist die Abgrenzung der die Insertionsstellen einfassenden heterochromatischen Segmente von den sogenannten euchromatischen

Enden leichter möglich. Es wur-

den Chromosomenlängen von 25-51 $\mu$ gemessen

VI. Beloperone guttata $(n=14)$.

Ein charakteristisches Merkmal für die Pachytänchromosomen von Beloperone ist die geringe Heterochromasie. Bevorzugt findet sich locker und in kleinen Portionen angeordnet ein Großteil des Heterochromatins an den Insertionsstellen und nur ganz geringe Mengen in den euchromatischen Chromosomenenden. Ihre Heterochromasie ist viel schwächer, als es zum Beispiel für die Chromsomen von Bellevalia beschrieben wurde (Oehlkers u. Eberle 1956). Deshalb ist die geringe Anfärbbarkeit und Spiralisation, sowie der fast kontinuierlich gleiche Chromosomendurchmesser verständlich. Ausser dem mit einem heterochromatischen Trabanten versehenen Nukleolenchromosom sind alle übrigen Chromosomen mit mittelständigem Heterochromatin beladen. In diesen Bereichen liegen auch median und submedian die Insertionsstellen. Die Chromosomenlängen betragen 29-46 $\mu$ (Abb. 10 und 11). 


\section{Diskussion}

\section{Die Strukturverhältnisse meiotischer Prophasechromosomen}

Über die beiden Chromatine, Eu- und Heterochromatin in ihrer ursprünglichen Abgrenzung (siehe Heitz 1935) wollen wir uns hier nicht befassen. Uns interessieren hier lediglich die Verhältnisse innerhalb eines bestimmten

Entwicklungsablaufes. Wir stützen uns im wesentlichen auf die im hiesigen Institut gezeigten

Ergebnisse bei Bellevalia (Oehlkers Eberle 1956), Paeonia (Eberle im Druck), Aloe (Eberle im

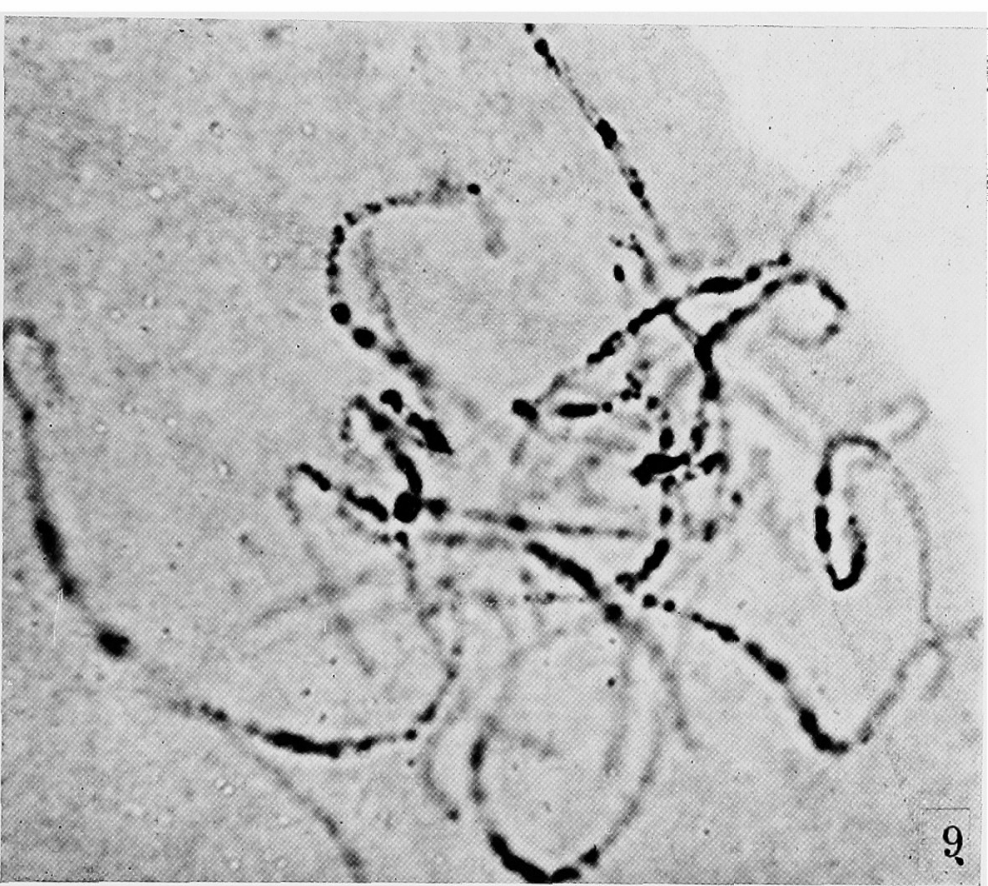

Abb. 9. Fittonia argyroneura. Pachytän. n=18; 2000:1.

Druck) und Compositen (Scherz im Druck). Danach ist die Struktur der meiotischen Prophasechromosomen nicht aus hetero- und euchromatischen Chromomeren zusammengesetzt, welche durch sogenannte Fibrillenstücke verbunden seien; vielmehr kann man am Chromosom nur hetero- und euchromatische Anteile unterscheiden (siehe auch Linnert 1955). Die Größe der genannten Segmente ist im Verlauf der meiotischen Prophase einem wechsel unterworfen, indem im wesentlichen nur die heterochromatischen Segmente bis zum Pachytän auf den zweifachen Durchmesser des Leptotäns anwachsen. Dieser Zuwachs ließ sich auf eine Nukleinsäurezunahme zurückführen (Oehlkers und Eberle 1956). Aufs engste sind diese Vorgänge verknüpft mit der meiotischen Spiralisation.

Auch bei den Acanthaceen finden sich Spiralisationsverhältnisse, wie sie bei Salvia, Gesneriaceen, Bellevalia, Paeonia, Aloe und Compositen beschrieben wurden. In sämtlichen Objekten sind die Pachytänchromosomen bereits stark spiralisiert. Und zwar sind der Spiralisationsgrad und die Anfärbung eng an die Menge des Heterochromatins gebunden, soda $\beta$ zum Beispiel bei dem sehr euchromatischen Objekt Beloperone nur eine geringe und lockere Spiralisation vorhanden ist; letztere ist vergleichsweise schwächer 


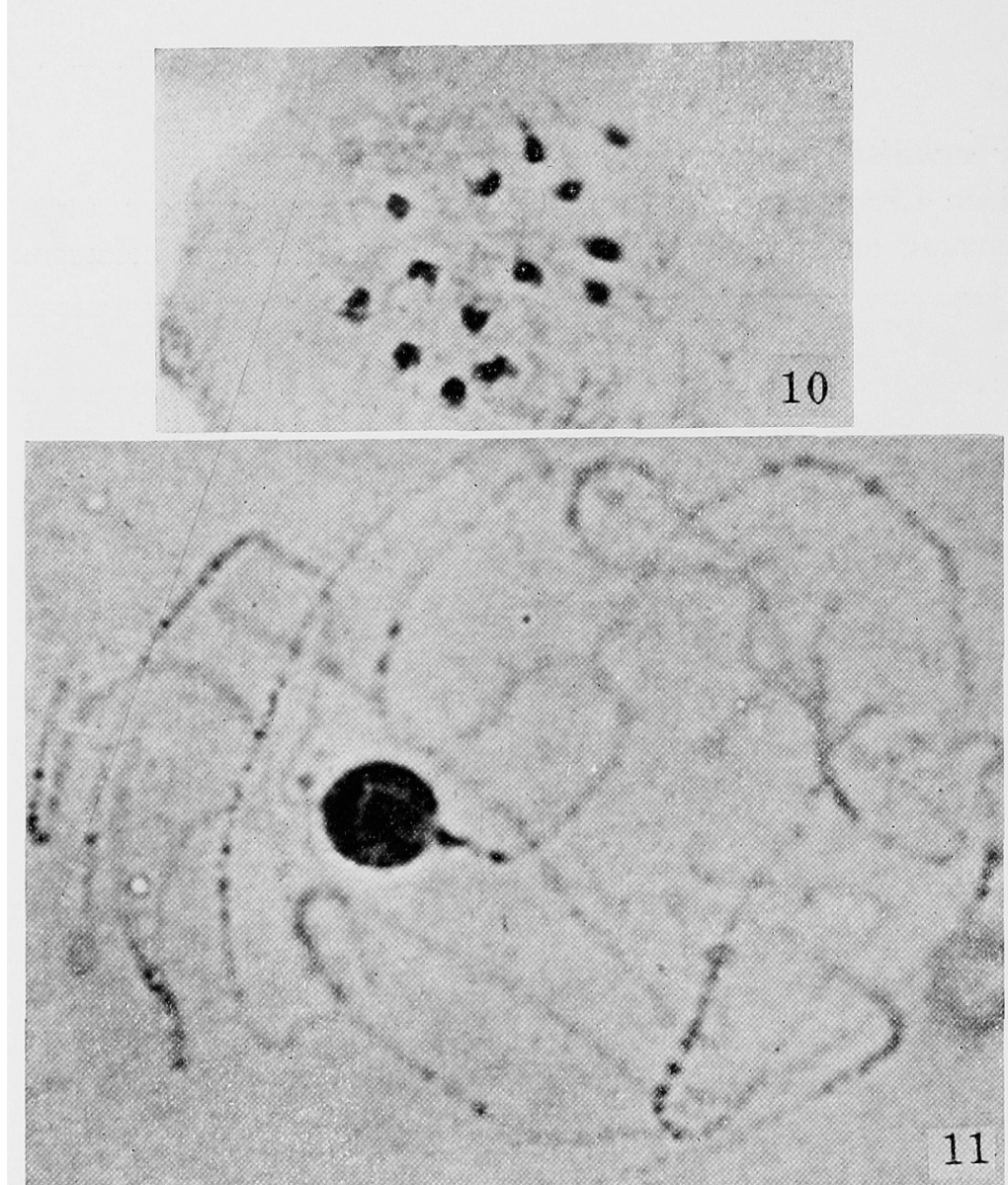

Abb. 10 u. 11. 10. Beloperone guttata. Teil einer An. I. $n=14 ; 2000: 1$. 11. Be'operone guttata. Pachytän. $n=14 ; 2000: 1$. als bei Bellevalia. Freilich verfügt Beloperone über eine lokale Anhäufung von Heterochromatin an der Insertionsregion. Wenn man den Beginn der meiotischen Spiralisation in Betracht zieht, so kann man die Acanthaceen mit Beloperone ebenfalls als Beispiel dafür anführen, daB die Spiralisation noch vor der Paarung beginnt. Diese erfolgt im späten Leptotän. Im frühen und mittleren Leptotän findet sich ein in hetero- und euchromatische Segmente gegliederter und völlig gestreckter Chromosomenfaden (Abb. 12).

Zwischen Leptotän und Pachytän findet sich auch die Antwort auf die Frage nach der Natur der Chromomeren. Im späten Leptotän zeigen die Chromosomenfäden eine feine Wellung, welche das ganze Chromosom erfaßt. Es werden also sowohl die hetero- als auch die euchromatischen Chromosomenanteile - und andere als die genannten Strukturelemente sind im Leptotänchromosom nicht erkennbar - in die entstehenden Chromosomenspiralen mit einbezogen. Gleichzeitig wird die Nukleinsäurebeladung insbesondere der heterochromatischen Segmente erhöht. Als Folge davon erscheinen spätestens im Pachytän runde und kugelige Gebilde, welche herkömmlich als Chromomeren bezeichnet werden. Zwischen diesen erscheinende, schwach gefärbte Chromosomenanteile geringeren Durchmessers können also keine sogenannten "Fibrillen" sein, sondern es sind die euchromatischen Chromosomenbestandteile. Je nach Spiralisationsgrad, Größe der heterochromatischen Segmente sowie dem regionalen Verhältnis von Hetero- und Euchromatin 
können sogenannte größere kompakte heterochromatische Chromosomensegmente erscheinen (Beispiele Fittonia, Thyrsacanthus). Wenn diese noch so dick sind beziehungsweise sich noch so dunkel angefärbt zeigen, darf dennoch nicht vergessen werden, da $\beta$ meist auch noch euchromatische Chromosomenanteile beteiligt sind, deren Existenz jedoch durch den Heterochromasie- und Spiralisationsgrad verdeckt wird.

Der Vergleich der Acanthaceen hinsichtlich

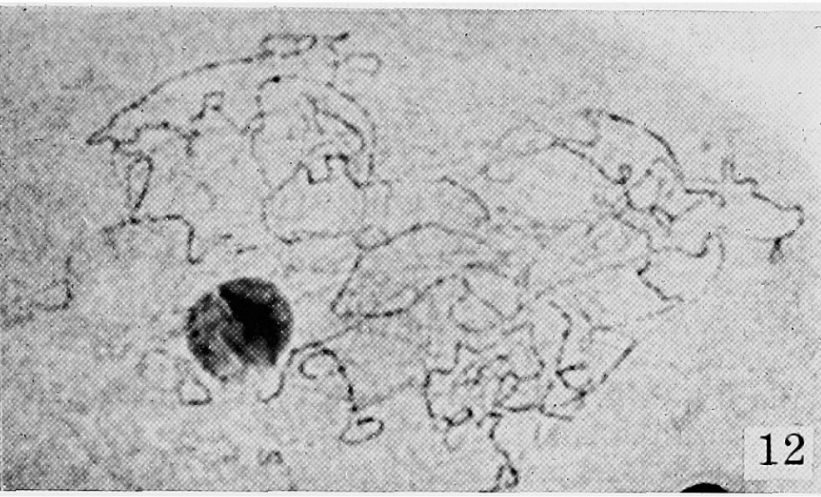

Abb. 12. Ruellia devosiana. Leptotän. Die maximal gestreckten Chromosomen zeigen eine Längsgliederung in hetero- und euchromatische Anteile. der Chromosomenstruktur mit anderen Tubifloren ergibt kein abweichendes Bild. Es liegen Chromatinverhältnisse vor, wie sie ebenso für Gesneriaceen (Oehlkers 1944, Eberle 1956), Digitalis (Linnert 1949), Solanum (Gottschalk 1954) und Salvia (Linnert 1955) beschrieben wurden.

\section{Zusammenfassung}

Es wurden die Chromosomenzahlen einer Reihe von Acanthaceen bestimmt. Bei einigen günstigen Objekten gelang es, die Struktur der Pachytänchromosomen zu beschreiben.

1. Die Haploidzahlen wurden festgestellt von Thunbergia alata $(n=9)$, Strobilanthes isophyllus $(n=10)$, Hemigraphis repanda $(n=14)$, Ruellia devosiana $(n=17)$, Aphelandra aurantiaca $(n=14)$ Aphelandra chamissoniana $(n=14)$, Peristrophe salicifolia $(n=21)$ Hyphoesthes phyllostachyi $(n=11-13)$, Thyrsacanthus rutilans $(n=21)$, Fittonia argyronetura $(n=18)$, Fittonia gigantea $(n=18)$, Fittonia verschaffeltii $(n=18)$, Justitia coccinea $(n=16)$, Beloperone guttata $(n=14)$.

2. Hinsichtlich der Struktur ihrer Pachytänchromosomen gehören die untersuchten Acanthaceen zu den sogenannten partiell-heterochromatischen Objekten.

3. Die geringste Heterochromasie findet sich bei Beloperone, die stärkste bei Aphelandra, Thyrsacanthus und Fittonia. Stets ist das Heterochromatin bevorzugt beiderseits der Insertionsstelle $z u$ finden. Letztere liegen meist median bis submedian.

4. Die Spiralisation der Pachytänchromosomen ist in allen Objekten vorhanden. Ihre Größe entspricht dem Maße der Heterochromasie. Stets finden sich auch in den sogenannten euchromatischen Chromosomenenden 
kleinere und größere Mengen von Heterochromatin.

5. Es gelang, die Pachytänchromosomen von Strobilanthes isophyllus und Beloperone guttata vollständig zu identifizieren. Bei anderen Objekten konnte ein Großteil der Chromosomen morphologisch erfaßt werden.

\section{Literatur}

Barton, D. W. 1950. Pachytene morphology of the tomato chromosome complement. Amer. J. Bot. $37: 639-643$.

Brown, S. W. 1949. The structure of meiotic behaviour of the differentiated chromosomes of tomato. Genetics 34: 437-461.

Darlington, C. A. and Janki A. E, 1945. Chromosome atlas of cultivated plants, G. Allen and Unwin Ltd., London.

Eberle, P. 1956. Cytologische Untersuchungen an Gesneriaceen I. Die Struktur der Pachytänchromosomen sowie eine Reihe neu bestimmter Chromosomenzahlen. Chromosoma 8: 285-316.

- 1956. Spiralen und Chromomeren in der frühen meiotischen Prophase von Paeonia tenuifolia. Chromosoma im Druck.

- 1956. Spiralen und Chromomeren in der frühen meiotischen Prophase von Aloe eru Berger. Chromosoma im Druck.

Gottschalk, W. 1951. Untersuchungen am Pachytän normaler und röntgenbestrahlter Pollenmutterzellen von Solanum lycopersicum. Chromosoma 4: 298-341.

- 1954. Die Chromosomenstruktur der Solanaceen unter Berücksichtigung phylogenetischer Fragestellungen. Chromosoma 6: 539-636.

Heitz, E. 1935. Chromosomenstruktur und Gene. Z. Vererbungslehre 70: 402-447.

Linnert G. 1948. Untersuchungen über die Cytologie polyploider Pflanzen I. Chromosoma 3: $328-356$.

- 1949. Untersuchungen über die Cytologie polyploider Pflanzen II. Chromosoma 3: 399417.

- 1955. Die Struktur der Pachytänchromosomen in Eu- und Heterochromatin und ihre Auswirkung auf die Chiasmabildung bei Salvia-Arten. Chromosoma 7: 90-128.

Moses, M. J. and J. H. Taylor 1955. Desoxypentose nucleic acid during microsporogenesis in Tradescantia. Exper. Cell Res. 9: 474-488.

Oehlkers, F. 1944. Cytologische und cytogenetische Untersuchungen an Streptocarpus. Z. Bot. 39: 113-153.

- 1956. Das Leben der Gewächse. Ein Lehrbuch der Botanik. Springer Verlag BerlinGöttingen-Heidelberg.

- und Eberle, P. 1956. Spiralen und Chromomeren in der Meiosis von Bellevalia romana. Chromosoma 8: 351-363.

Scherz, Ch. Die Chromosomenstruktur in der meiotischen Prophase einiger Composilen. Chromosoma im Druck.

Taylor, J. H. and MacMaster, R. D. 1954. Autoradiographic and microphotometric studies of desoxyribose nucleic acid during microgametogenesis in Lilium longiflorum. Chromosoma 6: 489-521. 